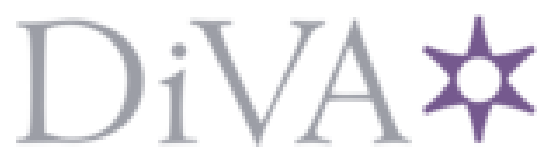

http://www.diva-portal.org

\title{
Postprint
}

This is the accepted version of a paper published in Journal of Micromechanics and Microengineering. This paper has been peer-reviewed but does not include the final publisher proof-corrections or journal pagination.

Citation for the original published paper (version of record):

Cruz, J. (2020)

Stable 3D Inertial Focusing by High Aspect Ratio Curved Microfluidics

Journal of Micromechanics and Microengineering

https://doi.org/10.1088/1361-6439/abcae7

Access to the published version may require subscription.

N.B. When citing this work, cite the original published paper.

Permanent link to this version:

http://urn.kb.se/resolve?urn=urn:nbn:se:uu:diva-427307 
ACCEPTED MANUSCRIPT

\section{Stable 3D Inertial Focusing by High Aspect Ratio Curved Microfluidics}

To cite this article before publication: Javier Cruz et al $2020 \mathrm{~J}$. Micromech. Microeng. in press https://doi.org/10.1088/1361-6439/abcae7

\section{Manuscript version: Accepted Manuscript}

Accepted Manuscript is "the version of the article accepted for publication including all changes made as a result of the peer review process, and which may also include the addition to the article by IOP Publishing of a header, an article ID, a cover sheet and/or an 'Accepted Manuscript' watermark, but excluding any other editing, typesetting or other changes made by IOP Publishing and/or its licensors"

This Accepted Manuscript is @ 2020 IOP Publishing Ltd.

During the embargo period (the 12 month period from the publication of the Version of Record of this article), the Accepted Manuscript is fully protected by copyright and cannot be reused or reposted elsewhere.

As the Version of Record of this article is going to be / has been published on a subscription basis, this Accepted Manuscript is available for reuse under a CC BY-NC-ND 3.0 licence after the 12 month embargo period.

After the embargo period, everyone is permitted to use copy and redistribute this article for non-commercial purposes only, provided that they adhere to all the terms of the licence https://creativecommons.org/licences/by-nc-nd/3.0

Although reasonable endeavours have been taken to obtain all necessary permissions from third parties to include their copyrighted content within this article, their full citation and copyright line may not be present in this Accepted Manuscript version. Before using any content from this article, please refer to the Version of Record on IOPscience once published for full citation and copyright details, as permissions will likely be required. All third party content is fully copyright protected, unless specifically stated otherwise in the figure caption in the Version of Record.

View the article online for updates and enhancements. 


\section{Stable 3D Inertial Focusing by High Aspect Ratio Curved Microfluidics}

Javier Cruz ${ }^{\mathrm{a}}$, Karin Hjort ${ }^{\mathrm{b}}$ and Klas Hjort ${ }^{\mathrm{a}}$

a. Engineering Sciences, Uppsala University, Ångström Laboratoriet, Uppsala, Sweden.

b. Dept. of Medical Biochemistry and Microbiology, Uppsala University, Uppsala, Sweden

Corresponding authors: Klas Hjort klas.hjort@angstrom.uu.se; Javier Cruz javier.cruz@angstrom.uu.se

Electronic Supplementary Information (ESI) available

\section{Abstract:}

Fine manipulation of particles is essential for the analysis of complex samples such as blood or environmental water, where rare particles of interest may be masked by millions of others. Inertial focusing is amongst the most promising techniques for this task, enabling label-free manipulation of particles with sub-micron resolution at very high flow rates. However, the phenomenon still remains difficult to predict due to the focus position shifting in tortuous ways as function of the channel geometry, flow rate and particle size. Here, we present a new line of microfluidics that exploit inertial focusing in High Aspect Ratio Curved (HARC) microchannels and overcome this limitation. Consisting of a single curved channel, HARC systems provide a highly predictable, single focus position near the centre of the inner wall, largely independent of the flow rate and particle size.

An explanation of the mechanism of migration and focus of particles, together with its governing equations, is provided based on simulations in COMSOL Multiphysics and experimental results. HARC microchannels built in silicon-glass were used for experimental validation, achieving a high quality, single focus position for a range of microparticles with sizes of $0.7-1 \mu \mathrm{m}$ and bacterial cells (Escherichia coli). The recovery of $1 \mu \mathrm{m}$ particles was $99.84 \%$ with a factor four in concentration.

With a stable focus position, we envision that HARC systems will bring the technology closer to implementation in laboratories for analysis of complex fluids with biological particles like cells and organelles.

\section{Introduction}

Microfluidics provide precise control and manipulation of fluids, enabling tasks that are normally carried out in standard laboratories to be performed on chip, such as mixing, separation and concentration of fluids, precise measurements and chemical analysis, to mention a few [1-3]. Miniaturization provides multiple advantages, as occupation of a small space, the need of tiny volumes of samples and reagents and very quick responses. The small scale of the systems also makes phenomena that do not happen in macro scale possible. This paper relates to one such unique effect; inertial focusing, a phenomenon that occurs in miniaturized fluidic channels and allows fine particle manipulation [4-6]. 
In microfluidics, surface forces usually dominate over body forces; viscosity dominates the system and inertial effects do not play a noteworthy role. Under such conditions, particles simply follow the streamlines of the carrier fluid, with diffusion as the only source of transversal displacement. There are, nevertheless, known techniques to manipulate such particles and make them move across streamlines in a controlled manner $[7,8]$. They are generally classified as active techniques if they require an external field (acoustic, electric, magnetic, etc.), or passive techniques if they only rely on the channel geometry and the fluid flow. These techniques make possible, for instance, the movement of particles from one fluid to another (e.g., for washing, reacting, mixing) or focusing, separation and concentration of a specific target.

Inertial focusing stands out amongst the particle manipulation techniques because it is passive, which makes the systems robust and simple to operate (the phenomenon arises simply by flowing the sample through the system if designed adequately), it is label free (it differentiates particles based on their size, geometry and deformability), the throughput is very high, and it can provide high focus quality and resolution [9].

Although operating the system is easy, the phenomenon of inertial focusing is complex. Yet, good progress has been made in the last decades, leading to devices that could process samples with circulating tumour cells $[10-12]$, bacteria $[9,13]$, exosomes [14], and other biological particles [6-8].

Briefly, in straight channels with circular cross section, randomly distributed particles migrate radially across the streamlines towards an equilibrium perimeter (EP). This migration is attributed to two main forces originated by the interaction of the fluid with the particle and the walls of the channel: the shear gradient induced lift force, which dominates at the centre of the channel and is directed towards the walls, and the wall-induced force, which dominates close to the walls and repels particles away from them. The combination of both is known as the net lift force [15] $\left(F_{L}\right)$ and becomes zero at the EP, where particles find equilibrium $[15,16]$. In non-circular cross-sections, this perimeter also exists, but $F_{L}$ has a minor component tangential to it (born from the asymmetry of the system) that gathers particles at the centre of the faces, Fig. 1A. These two effects, radial and lateral migration, overlap in time. However, the radial force is much stronger and the radial migration occurs much faster [17]. The difference is such that for practical reasons the radial migration can indeed be considered to occur first and be followed by the lateral one [18], as represented in Fig. 1B. Note that this implies that the component of the lift force that is orthogonal to the EP is very strong, while the tangential one is weak (in relative terms), which is critical for understanding systems with a secondary flow. Although more sophisticated and accurate calculations are available in the literature [19-21] a practical approximation for $F_{L}$ is:

$$
F_{L}=C_{L} \rho U_{m}^{2} a^{4} / W^{2}
$$

where $\rho$ is the density of the fluid, $U_{m}$ is the maximum flow velocity, $a$ is the size of the particle, $W$ is the smallest dimension of the cross section and $C_{L}$ is a coefficient that adjusts the value of the force as function of the Reynolds number ( $R e=\rho U_{m} W / \mu$; being $\mu$ the dynamic viscosity), the blockage ratio $k=a / W$, and the position of the particle in the channel $[15,18,22]$. 

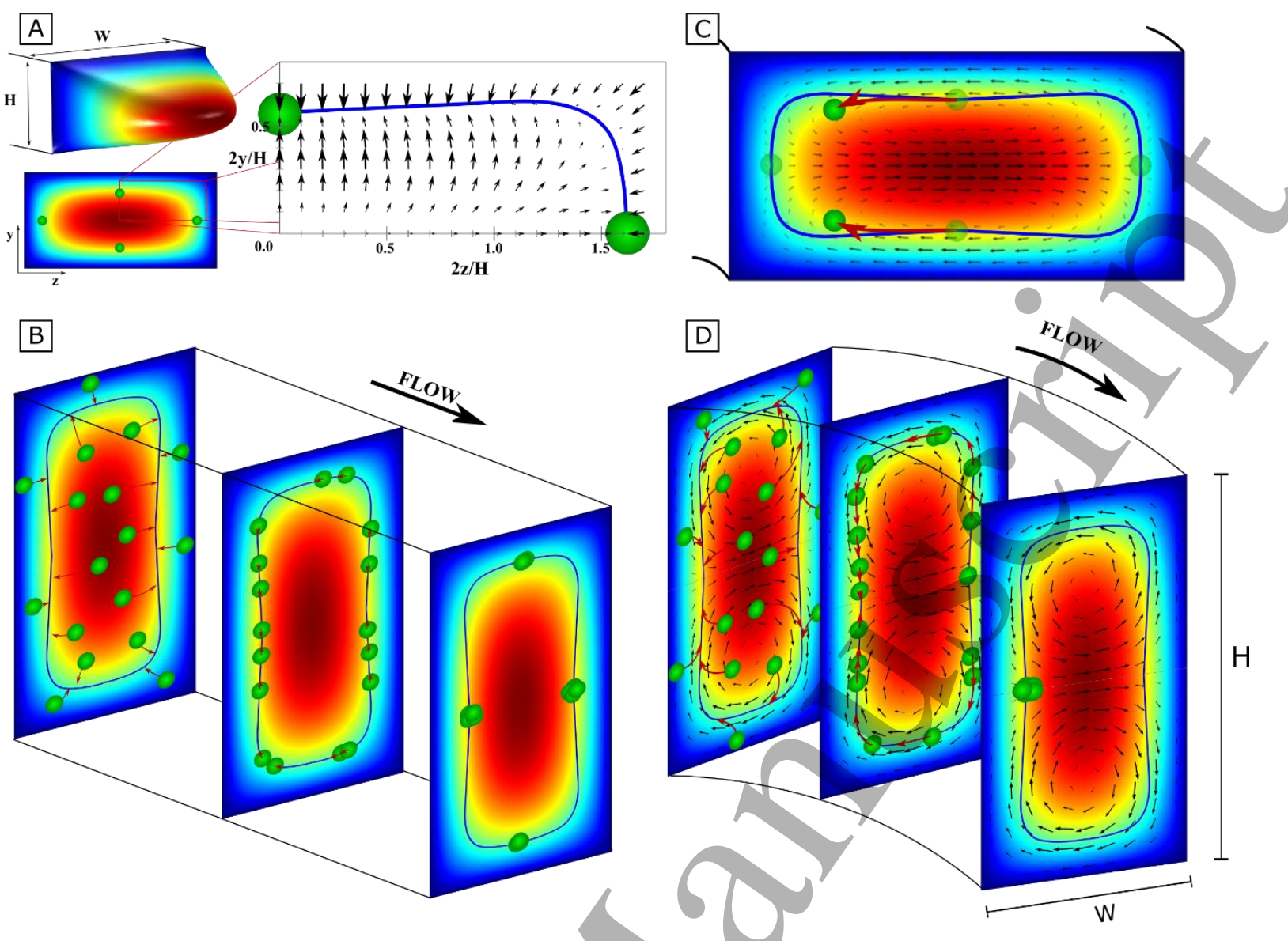

Figure 1. (A) Flow profile, lift force ( $F_{L}$; from Liu [22]; black arrows), equilibrium perimeter (EP; blue line) and focus positions in straight channels. (B) Sketch of the migration of particles in straight channels; particles first fall into the EP, to slowly slide along it towards four focus positions. (C) Modified focus positions in low aspect ratio curved channels by the secondary flow. (D) Sketch of the migration of particles in high aspect ratio curved (HARC) channels; particles fall to the EP and are collected around it by the secondary flow to focus in a single position by the inner wall.

As curvature is introduced in the system, a secondary flow $\left(U_{D}\right.$, named Dean flow; transversal to the main flow) appears, forming two symmetrical vortexes, Fig. 1C-D. The $A R$ defines the distribution of $U_{D}$ in a microchannel, while its magnitude scales as [23]:

$$
U_{D} \sim \frac{\rho}{\mu} \frac{U_{m}^{2} W^{2}}{R}
$$

where $R$ is the radius of curvature of the microchannel. These vortexes induce a drag to the particles parallel to their orbits $\left(F_{D}\right)$, modifying the distribution of forces and thus the focus positions. Assuming a Stokes drag, $F_{D}=3 \pi \mu a U_{D}^{*}$, where $U_{D}^{*}$ is the relative speed of the particle and the secondary flow; being $U_{D}^{*}=0$ if a particle freely follows the streamlines, and $U_{D}^{*}=U_{D}$ when a particle does not move, as is the case for particles stopped at the focus position. The introduction of a secondary flow has been reported to be advantageous; for instance, in low aspect ratio curved channels (with the aspect ratio defined as $A R=H / W$, being $H$ the height of the channel and the term "low" referring to $A R<1$ ). In said systems, the secondary flow leads to fewer focus positions (a single one or two symmetrical positions close to the inner wall that appear as one from top/bottom view) [9,24], Fig. 1C, and reduces the necessary channel length [25]. More details about of these systems and forces can be found in the literature $[4,5,20,26,27]$. 
Despite the attractive features of the technology and the plurality of biomedical applications demonstrated, inertial focusing is still nowadays limited in applicability outside research laboratories, with few successful examples such as Clear Cell FX1, a commercial product that separates circulating tumour cells from blood [28]. One of the reasons for this is the difficulty in predicting the focus position of the targets, which suffers large shifts in tortuous ways as a function of multiple parameters, the most critical being the particle size, flow rate and channel geometry [24].

Here, we explain and demonstrate for the first time inertial focusing in High Aspect Ratio Curved (HARC) microchannels; curved systems with rectangular cross section and $A R>1$ that overcome this limitation by providing a high quality, single focus position that is practically insensitive to particle size and largely invariant with the flow rate over a wide span, Fig. 1D. In addition to the excellent performance and predictability of the focus position, HARC systems consist of a single curved microchannel, bringing simplicity to the design. Simulations with COMSOL Multiphysics and experiments with microchannels built in silicon-glass were used to investigate the mechanism of migration and focusing of particles. A hypothesis is provided, together with two equations that define an upper and lower limit of flow rate where the focusing occurs.

Although they are fabricated very similarly to other microchannels for inertial focusing, inertial focusing in pure HARC microchannels has not yet been shown in the literature, while there are many studies with other curved geometries [25,29-31]. To the best of our knowledge, HARC sections have been used in two cases in relation to inertial focusing. First, to demonstrate that the secondary flow plays a major role in curved systems (i.e., that microparticles follow the secondary streamlines) [25]. Second, in a combination with straight channels, where the focus was induced by the straight segments and the HARC sections were used to iteratively bring particles out of the equilibrium position, achieving a single focus at the end [31]. In both cases, the secondary flow dominated over the lift force and was unable to focus particles.

We envision that HARC systems, with its stable 3D focus position, will bring the technology closer to implementation in analytical facilities for pre-treatment of complex fluid samples, such as blood or environmental water.

\section{Theory of HARC microchannels}

Figure 1D shows the secondary flow field in HARC microchannels and represents the migration of particles until the focus position. In HARC microchannels, similarly to in straight channels, there is a strong, radial component of $F_{L}$ induced by the main flow, which makes particles quickly migrate to the EP. The curvature induces a Dean flow that drags particles in the direction of its streamlines, as in other cross sections. The novelty of the HARC systems comes from the fact that the vortexes are mostly tangential to the EP, where $F_{L}$ almost vanishes, and therefore a small secondary flow is enough to make particles circulate around it. When particles reach the inner wall and move towards the central part, the vortexes turn and oppose the radial $F_{L}$. Provided that $F_{L}$ is strong enough, the horizontal component of $F_{D}$ is cancelled and the vertical one brings particles to a single position, on the symmetry line by the side of the inner wall. If, on the contrary, $F_{L}$ is not strong enough, particles cross over to the outer wall and keep circulating indefinitely. 
From this scenario, we conclude that a HARC microchannel must fulfil two conditions to focus the initially randomly distributed particles into the single focus position. The first, to have sufficient length for all particles to reach the focus position. The second, to induce a $F_{L}$ that surpasses $F_{D}$ for the targets of interest at the focus position. These two conditions are studied separately in the following sections.

Figure 2A shows COMSOL simulations of the secondary flow for $A R 2$ and 2.65, which are later used in the experimental validation. It can be seen that, in both cases, the vortexes will sweep particles around the EP and bring them to the aforementioned focus position. However, the strength of the Dean flow becomes weaker at the central part for the higher $A R$. Thus, higher $A R$ should be more convenient for focusing particles, as they would allow for higher flow rates, focusing of smaller particles, or (by a reduction in $R$ ) lowering the pressure.

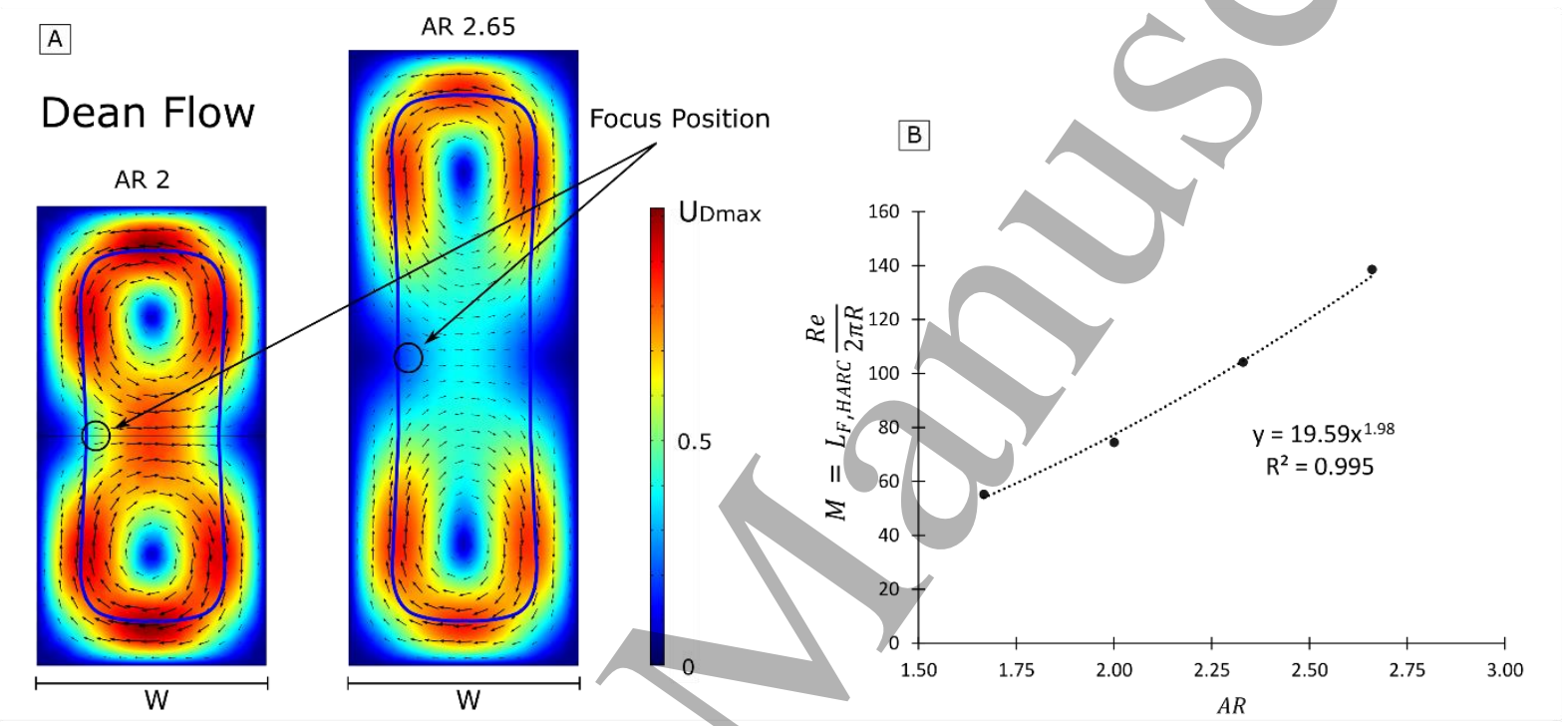

Figure 2. (A) Normalized secondary flows in HARC microchannels with $A R 2$ and 2.65 and similar, $U_{m}, W$ and $R$. The arrows represent the strength and direction, while the color represents only the strength. The blue line represents an approximation of the EP in straight channels. In both sections the secondary flow forms two vortexes, with the local strength strongly dependent on the $A R$. In particular, the strength at the focus position is much weaker for $A R 2.65$, while the velocity around the EP is comparable. (B) Coefficient $M$ as a function of $A R$, obtained by introducing in Eq. 3 values for $L_{F, H A R C}$ calculated with COMSOL for various $A R$, and fit for an analytical expression of $M=f(A R)$ (dotted line).

\section{Migration around the Equilibrium Perimeter}

An approximation to the necessary channel length for all particles to reach the concentration point $\left(L_{F, H A R C}\right)$ was calculated as the necessary channel length for the secondary flow to follow the EP from the central point at the outer wall to the central point at the inner one, to which we refer as a Dean Loop. We used COMSOL Multiphysics to calculate this channel length by integrating $\frac{U_{e p}}{U_{D e p}} d l$ over the EP (with $U_{e p}$ and $U_{D e p}$ being the local speed of the main and secondary flow and $d l$ an infinitesimal displacement on the EP, and the EP defined at a distance $0.25 \mathrm{~W}$ from the walls), reaching the expression:

$$
L_{F, H A R C} \approx \int_{E P} \frac{U_{e p}}{U_{D e p}} d l=M \frac{2 \pi R}{R e}
$$


with $M$ being a coefficient that strongly depends on $A R$. An analytical approximation to $M(M=$ $19.6 A R^{2}$ ) was obtained by fitting the calculations of the focus length obtained with COMSOL in microchannels with different $A R$, see Fig. 2B.

Expressing $L_{F, H A R C}$ as a number of loops $\left(N_{L}=\frac{L_{F, H A R C}}{2 \pi R}\right)$ :

$$
N_{L} \approx \frac{M}{R e}=\frac{19.6 A R^{2}}{R e}
$$

Equation 4 provides an approximation of the number of loops needed in the device to achieve focusing of particles. Small variations of the predicted value may be needed given that the equation was obtained for a generic EP and, depending on the particle size, the EP may be a bit closer or further from the walls. Note that, for a microchannel with a fixed number of loops, there will be a lower limit of $R e$ that will achieve focus. Indeed, re-shaping Eq. 4 provides the minimum flow rate $\left(Q_{\text {min }}\right)$ that will achieve a full Dean Loop for a microchannel with a given number of loops. For the case of water-based samples:

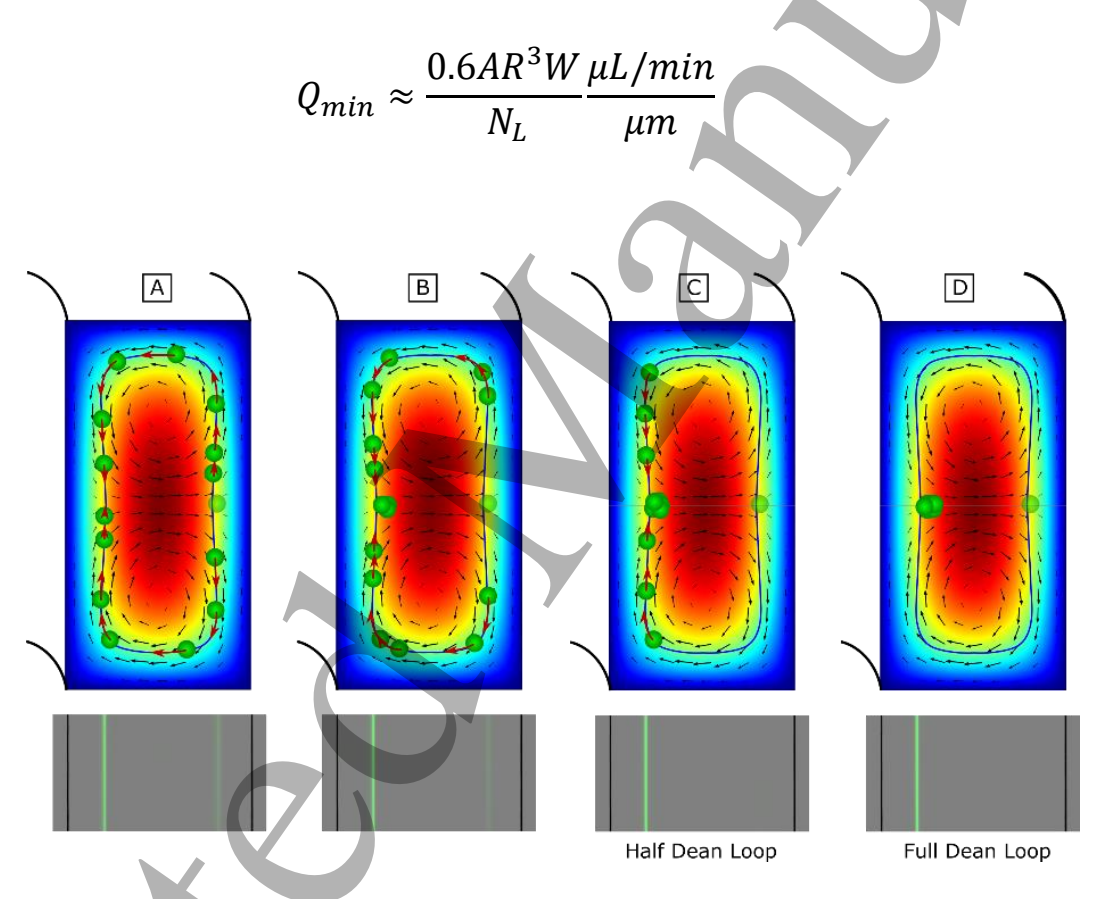

Figure 3. Different scenarios of the focusing process in HARC microchannels as the flow rate increases and its view under a fluorescent microscope at the expansion of the outlet (the microscope images correspond to the HARC system shown in Fig. 4). (A) Insufficient $Q$. (B) An increase in $Q$ makes more particles reach the inner wall; the intensity detected at the outer wall decreases. (C) $Q=\frac{1}{2} Q_{\text {min }}$, all particles have crossed to the side of the inner wall and form a plane; no intensity is detected at the outer wall. (D) $Q \geq Q_{\text {min }}$, all particles collapse into a single focus position.

Figure 3 shows a sketch of the migration around the EP in for different values of the flow rate and how the distribution of microparticles appears under a fluorescent microscope. At low values of $Q$, particles migrate to the EP and start following it. Two lines are seen under the fluorescent microscope, corresponding to the two vertical planes that contain most of the particles, Fig. 3A. As $Q$ is increased, more particles reach the inner wall and the intensity on the outer wall decreases, Fig. 3B. For $Q=\frac{1}{2} Q_{\text {min }}$, all particles have time to reach the side of the inner wall and form a plane, half a Dean Loop is completed and no intensity is detected at the outer wall, Fig. 3C. Finally, a further 
In practical applications where particles are focused, they reach the EP much faster than the collection around it, Fig. 1D. This conclusion is reached by assuming the scenario where the secondary flow is the fastest possible and still focus the particles; where $F_{D}=F_{L}$, as faster secondary flow will lead to particles surpassing the barrier of $F_{L}$. In this case, the radial migration by $F_{L}$ and the collection around the EP by the secondary flow will be, on average, equally fast, but the furthest distance that a particle needs to migrate radially until the EP is $\sim 0.3 W$, while the longest distance around the EP, for instance for $A R 2$, is $\sim 2 W$ (from the centre of the outer wall until the centre of the inner one). Particles thus reach the equilibrium perimeter, over which they slide almost freely following the secondary flow, at least $\sim 7$ times than what it takes for the collection to finish. Hence, the travel time for a particle to do a full Dean Loop is the limiting factor for the concentration and it can be used to estimate the necessary channel length for all particles to reach the focus position.

\section{Material and Methods}

\section{COMSOL Simulations}

Simulations were performed with COMSOL Multiphysics v.5.5. The 3D flow was solved using NavierStokes in curved microchannels extending a quarter of a loop with water as a fluid at room temperature. The analysis of the secondary flow was performed in a cross section at $2 / 3$ of the channel length from the inlet, ensuring a fully developed flow. To achieve a fine partition of the domain and minimize errors, the mesh was automatically generated and the maximum mesh size was set to $W / 30$

\section{Fabrication of the microchannels}

The microchannels were micromachined in silicon and sealed by anodically bonded glass, as it has been shown that such chips can work safely up to 200 bar [9]. A chromium mask from Micro 
Lithography Services Limited with $0.15 \mu \mathrm{m}$ CD was used. First, the microchannels were dry etched on silicon with photoresist (1813) as mask. Then, the wafer was cleaned and $0.5 \mu \mathrm{m}$ of Al were sputtered as an etch stop for the next step. Photolithography was done on the backside of the same wafer with a plastic mask from the same company ( $5 \mu \mathrm{m}$ resolution) and vias were dry etched at the inlets and outlets. The patterned wafer was cleaned together with a borosilicate wafer in Piranha solution for 20 minutes and then anodically bonded. Finally, the wafer was diced in small chips, to which glass capillaries were glued with epoxy (EPO-TEK ${ }^{\circledR}$ 302-3M).

\section{Design of the microchannels}

The microchannels comprised an inlet, a pillar section that served as a filter, two loops with similar radii, an expansion to allow a better visualization and one or more outlets, Fig. 4A. In order to include two loops with very similar $R$, the spiral consisted of 4 semi-circumferences with a consecutive increase in $R$ of $10 \mu \mathrm{m}$. The width was chosen as $6 \mu \mathrm{m}$ so that the smallest particle size in the experiments $(0.7 \mu \mathrm{m})$ fulfilled $k>0.1$, as with lower blockage ratios inertial focusing has been shown to be unfeasible [31]. Microchannels with $R$ of 300, 400 and $500 \mu \mathrm{m}$ were fabricated, with the lower number limited by the size of the inlet and filter, and the upper number limited by the increasing length of the channel with $R$, which leads to very high hydraulic resistances and would not allow for sufficient flow rates to fulfil Eq. 5 even at 200 bar, our practical pressure limitation. Two batches were fabricated, with depths of $12 \mu \mathrm{m}$ and $16 \mu \mathrm{m}$ ( $A R 2$ and 2.65, respectively).

\section{Experiments}

Fluorescent polystyrene particles $\left(0.70,0.79,0.92\right.$ and $1.0 \mu \mathrm{m}$, Thermo Scientific ${ }^{\mathrm{TM}}$ Fluoro-Max) were suspended in deionized water (with $0.1 \%$ of Triton $X$ to reduce agglomeration) in a concentration of $\sim 0.001$ vol\% and used to evaluate the focusing capabilities of the HARC systems with different particle sizes.

Escherichia coli MG1655 (E. coli, DA32838, in house strain collection) with a dTomato fluorescent tag (galK::cat-J23101-dTomato) was used to demonstrate the focusing capabilities with biological cells. The bacterial growth was initiated by inoculating a single colony from a LB (Lysogeny broth) plate into $1 \mathrm{ml} \mathrm{LB}$ medium. The bacterial culture was incubated at $37{ }^{\circ} \mathrm{C}$ with agitation (190 rpm) over night. The day after, $600 \mu \mathrm{L}$ of bacteria was transferred to $10.5 \mathrm{~mL}$ ice-cold $74 \%$ ethanol (final concentration $70 \%$ ) and incubated at $+4{ }^{\circ} \mathrm{C}$ for $2 \mathrm{~h}$. The cells were pelleted at $17000 \mathrm{~g}$ for $4 \mathrm{~min}$ at 4 ${ }^{\circ} \mathrm{C}$ and the supernatant was removed. The cell pellet was dissolved in $10 \mathrm{ml}$ sterile deionized water.

We used an HPLC pump (Waters, model 515) to run the samples with a read out of the pressure. The samples were loaded in a metallic tube with $5 \mathrm{~mL}$ capacity in-between the chip and the pump to avoid particles passing through the pump.

The evaluation of the microchannels was done with an inverted fluorescence microscope (Olympus IX73, equipped with a Orca-Flash 4.0 LT digital CMOS camera). Images were taken while increasing the pressure in steps of 10 bar. The analysis of the images was done with ImageJ at the expansion of the outlet.

To complement the optical evaluation, we collected the outlets of a chip ( 2 loops, $R 400 \mu \mathrm{m} ; 6 \times 16$ $\mu \mathrm{m}(W x H))$ for 10 minutes in Eppendorf tubes while running a sample with $1 \mu \mathrm{m}$ particles. The tubes were weighted to measure the amount of fluid going through each outlet, and a sample of $1 \mu \mathrm{L}$ from 
each tube was pipetted on a glass slide and allowed to dry ( 3 times each). An image of the resulting deposited particles was taken under the microscope and their number was counted with the tool "Find maxima" of ImageJ.

\section{Results and Discussion}

In an initial test, we found that the microchannels with $R 400 \mu \mathrm{m}$ were in the desired range. Smaller $R$ induced such a strong secondary flow that the microchannel was unable to focus all sizes in the set (0.7-1 $\mu \mathrm{m})$, and larger $R$ demanded higher pressures. Therefore, $R 400 \mu \mathrm{m}$ was chosen to validate the hypothesis about HARC systems.

Figure 4A shows an overview of a chip with $A R 2$ (2 loops; $R 400 \mu \mathrm{m} ; 6 \times 12 \mu \mathrm{m}(W x H)$ ) and a screenshot of the performance with $1 \mu \mathrm{m}$ polystyrene particles at $20 \mu \mathrm{L} / \mathrm{min}$. Fig. $4 \mathrm{~B}$ shows a compilation of the performance with the same particles in a range of flow rates and pressures. The experiments agreed with the hypothesis presented for the working mechanism; as predicted, particles focused with high quality at the inner wall. Interestingly and contrary to what happens in inertial focusing with other cross sections $[24,29,30]$, the focus position was practically insensitive to the flow rate, making the system more robust against possible drifts of the pump, which would normally lead to fluctuations in the focus positions and therefore diminished performances of the systems. The stability originates in the fact that the focus position lies at the symmetry line, where $F_{L}$ quickly grows from zero at $\sim 0.22 W$ (the EP) to its maximum value at $\sim 0.35 W$ from the inner wall [22]. With $F_{D}$ sharing the same direction (only horizontal), the particles are confined between these two points; particles focus close to the EP if $F_{L}$ dominates, and advance their position over the symmetry line towards the peak of $F_{L}$ as the secondary flow gains relevance. In other curved systems, both $F_{L}$ and $F_{D}$ have a vertical and a horizontal component at the equilibrium position, which leads to more complex shifts as their relative strength changes [9].

Furthermore, for this particular device, Eq. 5 predicts that one Dean Loop will be completed for 14 $\mu \mathrm{L} / \mathrm{min}\left(Q_{\min }\right)$, achieving the single focus position. In the experiments, after $\sim 7 \mu \mathrm{L} / \mathrm{min}\left(\equiv \frac{1}{2} Q_{\min }\right)$ no intensity was detected at the outer wall, corresponding to the situation of half Dean Loop, where all particles are on a plane by the side of the inner wall (Fig. $3 \mathrm{C}$ ), indicating that Eq. 5 is a good approximation. As $Q$ is increased, the plane is predicted to narrow down and collapse into a single line for $Q \geq Q_{\min }$ (Fig. 3D). However, based on a top view fluorescence image, we could not distinguish particles forming a plane or a single line and could not validate this hypothesis.

On the other hand, since $F_{L} \sim C_{L} U_{m}^{2}$ (with $C_{L}$ decreasing as $U_{m}$ increases [32-34]) and $F_{D} \sim U_{m}^{2}$ (see Eq. 1 and 2), $F_{L}$ does not grow as fast as $F_{D}$ as the flow rate increases. Therefore, for a given device, increasing the flow rate will eventually lead to $F_{D}$ surpassing $F_{L}$ and the device will lose the focusing capabilities. We refer to this upper limit as $Q_{\max }$, which may be calculated with Eq. 6 if $F_{L}$ is known. However, as of today, different studies point at different values of $F_{L}$ and no certain prediction could be done.

With our experiments, we validated the existence of the aforementioned $Q_{\max }$. At $Q>26 \mu \mathrm{L} / \mathrm{min}$, the microchannel lost its focusing capacities, Fig. 4B. At this stage, the secondary flow dominated at the central part for the given particle size and the particles went through the barrier of $F_{L}$, following the vortexes indefinitely. This indicates that $F_{D}$ increases faster with $U$ than $F_{L}$ or, in other words, that $C_{L}$ decays with $U$, which has been previously shown $[18,22]$. 
The focus was of high quality in the range $7-26 \mu \mathrm{L} / \mathrm{min}$; a range that is suitable to fractionate the sample in different outlets. On the other hand, for laser interrogation, where a single focus position is preferred, the estimated working range would be $14-26 \mu \mathrm{L} / \mathrm{min}$.

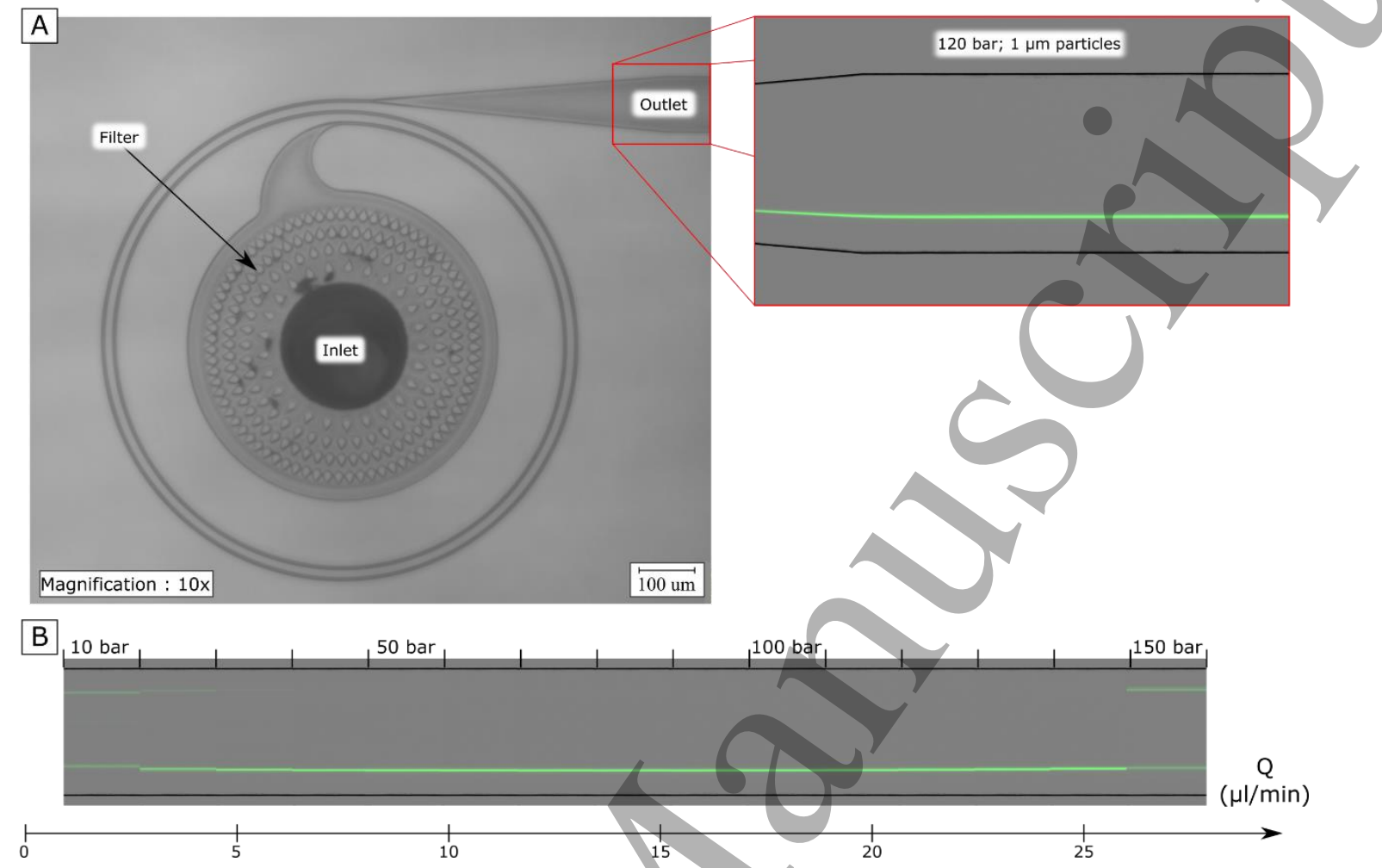

Figure 4. (A) Overview of a chip with 2 loops of HARC microchannels ( $A R 2$; 6x12 $\mu \mathrm{m}(W x H)$ and $R 400 \mu \mathrm{m})$ and a screenshot of the performance with $1 \mu \mathrm{m}$ polystyrene particles at $20 \mu \mathrm{L} / \mathrm{min}$. (B) Compilation of the performance with the same particles in a range of flow rates and pressures.

According to the COMSOL simulations, in $A R 2.65$ the collection around the EP has very similar speed, while the secondary flow at the focus position has half the strength of that in $A R 2$, Fig. $2 \mathrm{~A}$. Therefore, as mentioned before, a microchannel with $A R 2.65$ should be able to focus the same particles up to much higher flow rates. To validate this, we used the same design with an increase of $4 \mu \mathrm{m}$ in depth ( $A R$ 2.65, 2 loops; $R 400 \mu \mathrm{m} ; 6 \times 16 \mu \mathrm{m}(W x H))$ and compared the performance. The advantage of the higher AR was evident in the experiments, Fig. 5A. For $1 \mu \mathrm{m}$ particles, $Q_{\max }$ was more than double compared to in $A R 2(67 \mu \mathrm{L} / \mathrm{min}$ instead of $26 \mu \mathrm{L} / \mathrm{min})$. We also tested the channel with $0.70,0.79$ and $0.92 \mu \mathrm{m}$ particles, and found that all sizes focused close to the inner wall at a very similar position in the working range of flow rates, Fig. $5 \mathrm{~A}$. What is more, $Q_{\min }$ was independent of $a$ for the four particles used and well predicted by Eq. $5\left(Q_{\min }=33 \mu \mathrm{L} / \mathrm{min}\right)$, further supporting the fact that the collection around EP is the limiting factor in the focusing. On the other hand, we found $Q_{\max }$ to be strongly dependent on $a$ for the four sizes tested, which was expected since $F_{L} \sim a^{4}$ while $F_{D} \sim a$. Thus, stronger secondary flows are needed for larger particles to surpass the barrier of $F_{L}$.

Bacteria was used in the same device to evaluate the performance with biological particles, as these generally have different features than polymeric particles such as shape or rigidity. Figure 5B shows that $E$. coli, a rod-shaped bacteria, was focused with very high quality in a comparable manner to spherical polystyrene particles, proving the applicability of HARC systems to biological particles. The 
experiments agreed with our reported results with low aspect ratio microchannels, where $E$. coli behaved approximately as $1 \mu \mathrm{m}$ spherical particles [9].

To verify the quality of the results observed by optical imaging with the microscope, we ran a sample with $1 \mu \mathrm{m}$ particles at $33 \mu \mathrm{L} / \mathrm{min}\left(Q_{\min }\right)$ through a chip with the same focusing section and 3 outlets, see Fig. ESI 1. The fractionated sample from the outlet close to the inner wall contained $99.84 \%$ of the particles and $25 \%$ of the volume (factor four of concentration), the sample from the middle outlet contained no particles and $25 \%$ of the volume, and the one from the outlet close to the outer wall contained $0.16 \%$ of the particles and $50 \%$ of the volume, with an error of $\pm 0.03 \%$ at $95 \%$ level of confidence. The small fraction found at the outlet close to the outer wall likely corresponds to those particles that are initially on the symmetry line and do not follow any of the vortexes, as explained in section "Migration around the Equilibrium Perimeter".

With all particles focusing at a very similar position that remains stable with the flow rate, HARC systems overcome the main limitation of other curved systems exploiting inertial focusing, where the focus positions are different as function of particle size and vary with the flow rate $[24,29,30]$, making it difficult to tailor the technology for particular applications. However, while convenient for focusing, concentration of particles and purification of a fluid, HARC systems lose the capacity to separate particles by size, another of the main features of inertial focusing.

The experimental results and COMSOL simulations pointed at higher $A R$ being more convenient since they work with higher flow rates while demanding very similar pressures. The working range $\left(Q_{\min }<Q<Q_{\max }\right)$ was also wider for higher $A R$. However, from the COMSOL simulations, we estimate that the microchannels lose the focusing capacity for $A R$ much higher than those presented here $(A R \sim 4)$, as the secondary flow becomes very slow at the central regions and it is likely that it does not have the capacity to collect the particles, Fig. ESI 2. 


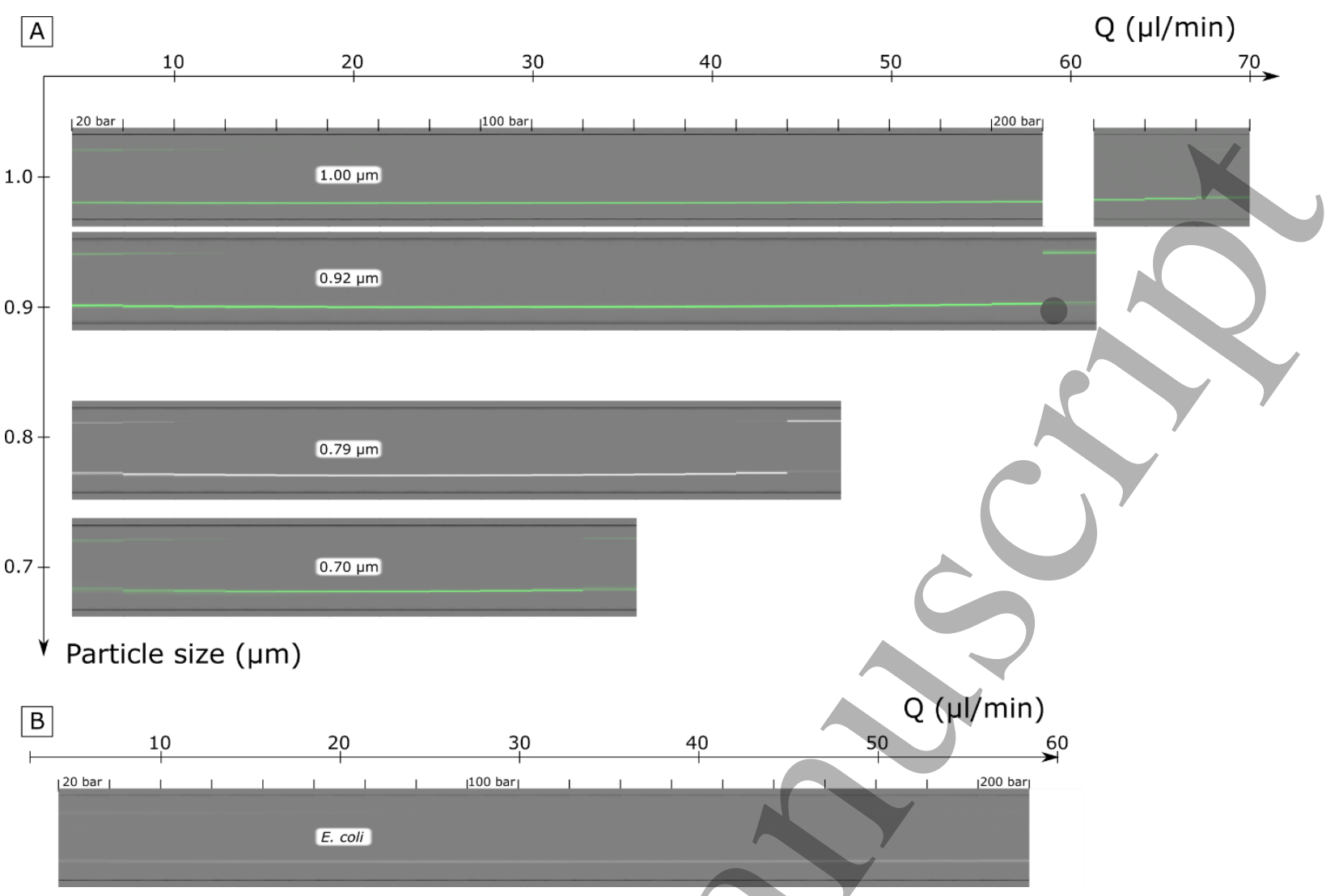

Figure 5. Compilation of the performance of a chip (2 loops of HARC microchannels $(A R 2.65 ; 6 \times 16 \mu \mathrm{m}(W x H)$ and $R 400$ $\mu \mathrm{m})$ in a range of flow rates and pressures. (A) Four particle sizes $(0.70,0.79,0.92$ and $1.0 \mu \mathrm{m})$. All particles focus in a line close to the inner wall at approx. $P>60$ bar $(Q>17 \mu \mathrm{L} / \mathrm{min})$. A strong relation is observed between the size of particle and $Q_{\max }$ (the flow rate where $F_{D}$ surpasses $F_{L}$ and particles are not focused any longer). The value of $Q_{\max }$ for $1 \mu \mathrm{m}$ particles demanded more than 200 bar; it was observed in a similar device with a single loop, which demanded half of the pressure. (B) Escherichia coli cells, demonstrating the applicability of HARC microchannels to biological particles.

With a focus position independent of the particle size, HARC systems are ideal for purification of fluids and concentration of particles in a range of sizes, e.g. a population of bacteria or of several different species. With the focus position being stable within a range of flow rate, HARC systems are robust and non-dependent on high precision pumps/control systems. As no sheath is needed, HARC systems may also be advantageous for technologies that require a focused sample like Flow Cytometry, bringing simplicity to the operation, saving reagents and multiplying the throughput.

In this work, the working range of HARC systems was identified and described. An expression to calculate its lower limit, which relates to the minimum channel length for particles to reach the single focus position, was provided and verified experimentally. Further investigation is needed to obtain an expression for the upper limit, over which particles remain unfocused.

\section{Conclusion}

With this work, we introduce inertial focusing in High Aspect Ratio Curved (HARC) microchannels. These novel systems are easy to design and offer very attractive properties, such as a single focus position, largely independent of the flow rate and particle size. Both theory and experiments showed a trend where microchannels with higher aspect ratios offer better performance. The higher the aspect ratio, the weaker the secondary flow becomes at the focus position, which translates into 
higher flow rates until the secondary flow dominates for a fixed particle size, suitability to focus smaller particles or a possible reduction in the pressure demand. However, a too high aspect ratio $(A R \sim 4)$ has such a low secondary flow at the central part that it can be expected to lose the focusing capabilities.

HARC microchannels overcome one major limitation of other systems exploiting inertial focusing, in which the focus position of the targets shifts in tortuous ways as function of the flow rate, particle size and channel geometry. Given the outstanding performances of inertial focusing in the field of particle manipulation, HARC systems can be expected to be implemented in laboratories for preparation of complex samples with particles.

\section{References}

[1] Mark D, Haeberle S, Roth G, Von Stetten F and Zengerle R 2010 Microfluidic lab-on-a-chip platforms: Requirements, characteristics and applications Chem. Soc. Rev. 39 1153-82

[2] Sackmann E K, Fulton A L and Beebe D J 2014 The present and future role of microfluidics in biomedical research Nature 507 181-9

[3] Convery N and Gadegaard N 201930 years of microfluidics Micro Nano Eng. 2 76-91

[4] Martel J M and Toner M 2014 Inertial Focusing in Microfluidics Annu. Rev. Biomed. Eng. 16 371-96

[5] Zhang J, Yan S, Yuan D, Alici G, Nguyen N T, Ebrahimi Warkiani M and Li W 2016 Fundamentals and applications of inertial microfluidics: A review Lab Chip 16 10-34

[6] Gou Y, Jia Y, Wang P and Sun C 2018 Progress of inertial microfluidics in principle and application Sensors 181762

[7] Zhang T, Hong Z Y, Tang S Y, Li W, Inglis D W, Hosokawa Y, Yalikun Y and Li M 2020 Focusing of sub-micrometer particles in microfluidic devices Lab Chip 20 35-53

[8] Zhou J, Mukherjee P, Gao H, Luan Q and Papautsky I 2019 Label-free microfluidic sorting of microparticles APL Bioeng. 3041504

[9] Cruz J, Graells T, Walldén M and Hjort K 2019 Lab on a Chip PAPER Inertial focusing with submicron resolution for separation of bacteria + Lab Chip 19 1257-66

[10] Warkiani M E, Guan G, Luan K B, Lee W C, Bhagat A A S, Kant Chaudhuri P, Tan D S W, Lim W $T$, Lee S C, Chen P CY, Lim C T and Han J 2014 Slanted spiral microfluidics for the ultra-fast, label-free isolation of circulating tumor cells Lab Chip 14 128-37

[11] Warkiani M E brahim., Khoo B L ua., Wu L, Tay A K a. P, Bhagat A A sga. S, Han J and Lim C T ec. 2016 Ultra-fast, label-free isolation of circulating tumor cells from blood using spiral microfluidics Nat. Protoc. 11 134-48

[12] Zhou J, Kulasinghe A, Bogseth A, O'Byrne K, Punyadeera C and Papautsky I 2019 Isolation of circulating tumor cells in non-small-cell-lung-cancer patients using a multi-flow microfluidic channel Microsystems Nanoeng. 58 
[13] Cruz J, Hooshmand Zadeh S, Graells T, Andersson M, Malmström J, Wu Z G G and Hjort K 2017 High pressure inertial focusing for separating and concentrating bacteria at high throughput J. Micromechanics Microengineering 27084001

[14] Liu C, Guo J, Tian F, Yang N, Yan F, Ding Y, Wei J, Hu G, Nie G and Sun J 2017 Field-Free Isolation of Exosomes from Extracellular Vesicles by Microfluidic Viscoelastic Flows ACS Nano $116968-76$

[15] Ho B P and Leal L G 1974 Inertial migration of rigid spheres in two-dimensional unidirectional flows J. Fluid Mech. 65365

[16] Matas J P, Morris J F and Guazzelli E 2004 Lateral forces on a spehere 59 59-70

[17] Choi Y S, Seo K W and Lee S J 2011 Lateral and cross-lateral focusing of spherical particles in a square microchannel Lab Chip 11 460-5

[18] Zhou J and Papautsky I 2013 Fundamentals of inertial focusing in microchannels Lab Chip 13 1121-32

[19] Liu C, Xue C, Sun J and Hu G 2016 A generalized formula for inertial lift on a sphere in microchannels Lab Chip 16 884-92

[20] Hood K, Lee S and Roper M 2015 Inertial migration of a rigid sphere in three-dimensional Poiseuille flow J. Fluid Mech. 765 452-79

[21] Asmolov E S, Dubov A L, Nizkaya T V., Harting J and Vinogradova O I 2018 Inertial focusing of finite-size particles in microchannels J. Fluid Mech. 840 613-30

[22] Liu C, Hu G, Jiang X and Sun J 2015 Inertial focusing of spherical particles in rectangular microchannels over a wide range of Reynolds numbers Lab Chip 15 1168-77

[23] Squires T M and Quake S R 2005 Microfluidics: Fluid physics at the nanoliter scale Rev. Mod. Phys. 77 977-1026

[24] Martel J M and Toner M 2013 Particle focusing in curved microfluidic channels Sci. Rep. 3 1-8

[25] Gossett D R and Di Carlo D 2009 Particle focusing mechanisms in curving confined flows Anal. Chem. 81 8459-65

[26] Harding B, Stokes Y M and Bertozzi A L 2019 Effect of inertial lift on a spherical particle suspended in flow through a curved duct J. Fluid Mech. 875 1-43

[27] Stoecklein D and Di Carlo D 2019 Nonlinear Microfluidics Anal. Chem. 91 296-314

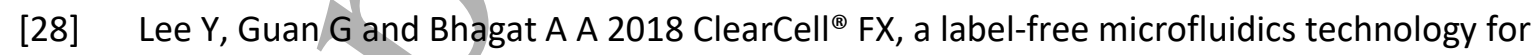
enrichment of viable circulating tumor cells Cytom. Part A 93 1251-4

[29] Zhao Q, Yuan D, Zhang J and Li W 2020 A review of secondary flow in inertial microfluidics Micromachines 11 1-23

[30] Chung A J 2019 A Minireview on Inertial Microfluidics Fundamentals: Inertial Particle Focusing and Secondary Flow Biochip J. 13 53-63

[31] Zhang Y, Zhang J, Tang F, Li W and Wang X 2018 Design of a Single-Layer Microchannel for Continuous Sheathless Single-Stream Particle Inertial Focusing Anal. Chem. 90 1786-94 
[32] Di Carlo D, Irimia D, Tompkins R G and Toner M 2007 Continuous inertial focusing, ordering, and separation of particles in microchannels Proc. Natl. Acad. Sci. 104 18892-7

[33] Schonberg J A and Hinch E J 1989 Inertial migration of a sphere in Poiseuille flow J. Fluid Mech. 203 517-24

[34] Asmolov E S 1999 The inertial lift on a spherical particle in a plane poiseuille flow at large channel Reynolds number J. Fluid Mech. 381 63-87

[35] Matas J P, Morris J F and Guazzelli É 2004 Inertial migration of rigid spherical particles in Poiseuille flow J. Fluid Mech. 515 171-95 